\title{
CENTRALIZERS OF TRANSITIVE PERMUTATION GROUPS AND APPLICATIONS TO GALOIS THEORY
}

\author{
CHAD AWTREY, NAKHILA MISTRY, AND NICOLE SOLTZ
}

\begin{abstract}
Let $f(x)$ be an irreducible polynomial of degree $n$ defined over a field $F$, and let $G$ be the Galois group of $f$, identified as a transitive subgroup of $S_{n}$. Let $K / F$ be the stem field of $f$. We show the automorphism group of $K / F$ is isomorphic to the centralizer of $G$ in $S_{n}$. We include two applications to computing Galois groups; one in the case $F$ is the rational numbers, the other when $F$ is the 5 -adic numbers.
\end{abstract}

\section{INTRODUCTION}

The work of 19th century mathematician Evariste Galois shows it is possible to associate a group structure to a polynomial's roots. This group, called the polynomial's Galois group, is a collection of permutations of the roots that encodes much arithmetic information concerning the polynomial. For example, the polynomial is solvable by radicals if and only if its Galois group is solvable (see for example [4, p. 628]). Recall that a polynomial is said to be solvable by radicals if its roots can be expressed using the following three items: (1) the polynomial's coefficients, (2) the four basic arithmetic operations $(+,-, \times, \div)$, and (3) radicals (square roots, cube roots, etc.).

An important problem in computational algebra therefore is to determine the Galois group of a polynomial $f(x)$ of degree $n$ defined over a field $F$. Most practical methods for computing Galois groups focus on factoring what are known as resolvent polynomials $[5,13,14]$. These are polynomials that define subfields of the splitting field of $f$. But the resolvent method can be computationally expensive, since resolvent polynomials generally have large degree (which makes factoring difficult) and/or they are formed using complex or $p$-adic approximations (this requires high precision for proven results). Implementations of these methods over $\mathbf{Q}$ can be found in many software programs, including but not limited to GAP [6] and PARI/GP [11]. We note that no general implementations exist for computing Galois groups over the field of $p$-adic numbers $\mathbf{Q}_{p}$. 


\section{CENTRALIZERS OF TRANSITIVE PERMUTATION GROUPS}

In this paper, we present a simple alternative to the resolvent method. Our approach is based on the centralizer of the Galois group in $S_{n}$ (the symmetric group of degree $n$ ). After providing a brief overview in Section 2 of the basic notation and terminology related to the Fundamental Theorem of Galois Theory, Section 3 contains a proof that the centralizer of the Galois group in $S_{n}$ of an irreducible polynomial of degree $n$ is isomorphic to the automorphism group of the polynomial's stem field (cf. Theorem 3.6).

Our final two sections provide applications of Theorem 3.6 to computing Galois groups. Section 4 gives an algorithm for computing Galois groups of irreducible quartic polynomials over arbitrary base fields (of characteristic $\neq 2$ ). This algorithm is an improvement over the traditional methods that use resolvent polynomials $[8,3]$. Section 5 gives a complete classification of all isomorphism classes of degree 15 extensions of the 5 -adic numbers whose Galois group is isomorphic to a direct product of the form $\mathrm{H} \times \mathrm{C}_{3}$ where $H$ is a solvable transitive subgroup of $S_{5}$, and $C_{3}$ is the cyclic group of order 3 . Note, there are only finitely many such isomorphism classes [9, p. 54]. This extends previous research on classifying finite extensions of $\mathbf{Q}_{p} ;$ see $[1,2]$ for the most recent results in this area.

\section{Definitions}

In this section we provide basic notation and terminology related to field extensions and their automorphism groups for the purpose of introducing the Fundamental Theorem of Galois Theory. Let $F$ be a field and let $\bar{F}$ be a fixed algebraic closure. Let $f(x) \in F[x]$ be an irreducible polynomial of degree $n$ with roots $\alpha_{1}, \ldots, \alpha_{n} \in \bar{F}$. Let $K$ be the stem field of $f$; that is, $K=F\left(\alpha_{1}\right)$.

Then it is straight forward to verify that $K$ is a vector space over $F$ with the set $\left\{1, \alpha, \ldots, \alpha^{-1}\right\}$ serving as a basis. Since the dimension of $K$ as an $F$-vector space is $n$, we say $K / F$ is an extension field of degree $n$ and we write $[K: F]=n$.

We are interested primarily in automorphisms of $K / F$, which are field isomorphisms from $K$ to itself that restrict to the identity function on $F$.

Definition 2.1. An automorphism of $K / F$ is a mapping $\sigma: K \rightarrow K$ such that,

(1) $\sigma$ is bijective,

(2) $\sigma(x+y)=\sigma(x)+\sigma(y)$ for all $x, y \in K$,

(3) $\sigma(x y)=\sigma(x) \sigma(y)$ for all $x, y \in K$,

(4) $\sigma(a)=a$ for all $a \in F$.

The collection of all automorphisms of $K / F$ is denoted $\operatorname{Aut}(K / F)$.

The automorphisms of $K / F$ form a group under function composition. Since automorphisms are field isomorphisms and since a basis for $K / F$

MISSOURI J. OF MATH. SCI., FALL 2015 


\section{AWTREY, N. MISTRY, AND N. SOLTZ}

consists of powers of the one root $\alpha_{1}$, it follows that each automorphism is completely determined by where it sends $\alpha_{1}$. Furthermore, since automorphisms act like the identity function on $F$, we have $0=\sigma(0)=\sigma\left(f\left(\alpha_{1}\right)\right)=$ $f\left(\sigma\left(\alpha_{1}\right)\right)$ for each $\sigma \in \operatorname{Aut}(K / F)$. This shows that automorphisms send $\alpha_{1}$ to some other root of $f$ that lies in $K$. Therefore, the automorphisms of $K / F$ are in one-to-one correspondence to the roots of $f$ that lie in $K$.

Definition 2.2. If $K / F$ contains all $n$ roots of $f$, then we say $K / F$ is a Galois extension. In this case, we call $\operatorname{Aut}(K / F)$ the Galois group of $f$. If $K / F$ contains fewer than $n$ roots of $f$, the Galois group of $f$ is defined to be $\operatorname{Aut}\left(K^{g} / F\right)$ where $K^{g}$ is the splitting field of $f$, the smallest subfield of $\bar{F}$ that contains $F$ and all $n$ roots of $f$.

The next result is the Fundamental Theorem of Galois Theory, which gives a bijection between the subfields of $K / F$ and the subgroups of $\operatorname{Aut}(K / F)$ when $K / F$ is a Galois extension (see [4, p. 574]).

Theorem 2.3 (Fundamental Theorem of Galois Theory). Let $K / F$ be a Galois extension with Galois group $G$. Let $E$ be a subfield of $K / F, H$ a subgroup of $G$, and $K^{H}$ the collection of elements in $K$ fixed by all the elements of $H$ (called the fixed field of $H$ ). That is, $K^{H}=\{k \in K: \sigma(k)=$ $k$ for all $\sigma \in H\}$.

(1) $K^{H}$ is a subfield of $K / F$.

(2) The maps $E \mapsto A u t(K / E)$ and $H \mapsto K^{H}$ are inverses of each other.

(3) $[K: E]=\# H$ and $[E: F]=[G: H]$ (the index of $H$ in $G$ ).

(4) $K / E$ is a Galois extension with Galois group $H$.

(5) $E / F$ is a Galois extension if and only if $H$ is normal in $G$. In this case, $\operatorname{Aut}(E / F) \simeq G / H$.

We now prove several facts about subfields of $K$ and subgroups of $\operatorname{Aut}(K / F)$. The first considers conjugate fields of a subfield $E$ of $K / F$. For $\sigma \in \operatorname{Aut}(K / F)$ a subfield $E$ of $K / F$, the image $\sigma(E)$ of $E$ under $\sigma$ is called a conjugate field of $E$. The next result shows that conjugate fields correspond to conjugate subgroups.

Proposition 2.4. Let $K / F$ be a Galois extension with Galois group $G$. Let $H$ be a subgroup of $G$ and let $E$ be the fixed field of $H$. For $\sigma \in G$, the subgroup fixing $\sigma(E)$ is $\sigma H \sigma^{-1}$.

Proof. First we show $\sigma H \sigma^{-1}$ fixes $\sigma(E)$. Let $\sigma h \sigma^{-1} \in \sigma H \sigma^{-1}$ and let $\sigma(x) \in \sigma(E)$. Then $\sigma h \sigma^{-1}(\sigma(x))=\sigma h(x)=\sigma(x)$, as desired.

Next we show that every element that fixes $\sigma(E)$ belongs to $\sigma H \sigma^{-1}$. Suppose $\tau \in G$ fixes $\sigma(E)$. Then for all $x \in E, \tau(\sigma(x))=\sigma(x)$. Thus, $\sigma^{-1} \tau \sigma(x)=x$. Thus, $\sigma^{-1} \tau \sigma$ fixes $E$, which implies $\sigma^{-1} \tau \sigma \in H$. Thus, $\tau \in \sigma H \sigma^{-1}$, as desired. 


\section{CENTRALIZERS OF TRANSITIVE PERMUTATION GROUPS}

Proposition 2.5. Let $K / F$ be a Galois extension with Galois group $G$. Let $H$ be a subgroup of $G$ and let $E$ be the fixed field of $H$. Let $\sigma, \tau \in G$. Then $\sigma(x)=\tau(x)$ for all $x \in E$ if and only if $\tau \in \sigma H$.

Proof. Let $\sigma, \tau \in G$ and suppose $\sigma(x)=\tau(x)$ for all $x \in E$. Then $x=$ $\sigma^{-1} \tau(x)$ for all $x \in E$. Thus, $\sigma^{-1} \tau$ fixes $E$, which is true if and only if $\sigma^{-1} \tau \in H$. This is true if and only if $\tau \in \sigma H$, as desired.

Proposition 2.6. Let $K / F$ be a Galois extension with Galois group $G$. Let $H$ be a subgroup of $G$ and let $E$ be the fixed field of $H$. Then Aut $(E / F) \simeq$ $N(H) / H$, where $N(H)$ is the normalizer of $H$ in $G$.

Proof. First we note that every element in $\operatorname{Aut}(E / F)$ corresponds to some restriction to $E$ of an element $\sigma \in G$ [4, p. 575]. Such an element $\sigma$ will restrict to an automorphism of $E / F$ precisely when $\sigma(E)=E$. By Proposition 2.4, these are restrictions of elements in the normalizer $N(H)$ of $H$ in $G$. By Proposition 2.5, the elements in $N(H)$ that restrict to distinct functions on $E$ correspond to the cosets of $H$ in $N(H)$. Therefore $\operatorname{Aut}(E / F)$ is isomorphic to the quotient group $N(H) / H$, as desired.

\section{Centralizers and Automorphism Groups}

In this section, we prove that the automorphism group of the stem field of an irreducible degree $n$ polynomial $f(x)$ defined over a field $F$ is isomorphic to the centralizer of its Galois group in $S_{n}$. Our proof involves analyzing two group actions on suitably chosen cosets of the Galois group. We begin with preliminary definitions and results.

Definition 3.1. Let $G$ be a group with identity element $e$, and let $X$ be a set. A group action of $G$ on $X$ is a function from $G \times X$ to $X$ where the image of $(g, x)$, denoted by $g \cdot x$, satisfies the following two properties:

(1) $e \cdot x=x$ for all $x \in X$

(2) $g \cdot(h \cdot x)=(g h) \cdot x$ for all $x \in X$ and all $g, h \in G$

For each $g \in G$, we can define a function $\sigma_{g}: X \rightarrow X$ by $\sigma_{g}(x)=g \cdot x$. It is straightforward to verify that each $\sigma_{g}$ has the following properties.

- $\sigma_{g}$ is a permutation, hence, $\sigma_{g} \in S_{X}$.

- $\sigma_{g} \sigma_{h}=\sigma_{g h}$.

- $\sigma_{e}=\mathrm{id}_{X}$.

- $\sigma_{g}^{-1}=\sigma_{g^{-1}}$.

- The function $\phi: G \rightarrow S_{X}$ defined by $\phi(g)=\sigma_{g}$ is a homomorphism. The function $\phi$ is called the permutation representation of $G$ acting on $X$.

Now we consider a group $G$ acting on left cosets of some subgroup $H \leq$ $G$.

MISSOURI J. OF MATH. SCI., FALL 2015 


\section{AWTREY, N. MISTRY, AND N. SOLTZ}

Proposition 3.2. Let $G$ be a group with identity element e, and let $H$ be a subgroup of $G$. Let $L$ be the set of left cosets of $H$ in $G$. Then $G$ acts on $L$ by left multiplication; i.e., for each $a \in G$,

$$
g \cdot(a H)=(g a) H,
$$

defines a group action of $G$ on $L$. Let $\lambda: G \rightarrow S_{L}$ be the permutation representation of this action. Then

$$
\operatorname{Ker} \lambda=\bigcap_{g \in G} g H g^{-1} .
$$

Proof. That $G$ defines a group action on $L$ follows directly from the two defining properties of group actions. Let $\lambda: G \rightarrow S_{L}$ be the permutation representation of this action, so that $\lambda(g)=\sigma_{g}$ where $\sigma_{g}(a H)=g a H$. Then we have,

$$
\begin{aligned}
\text { Ker } \lambda & =\left\{g \in G: \sigma_{g}=\operatorname{id}_{L}\right\} \\
& =\left\{g \in G: \sigma_{g}(a H)=a H \text { for all } a \in G\right\} \\
& =\{g \in G: g a H=a H \text { for all } a \in G\} \\
& =\left\{g \in G: a^{-1} g a \in H \text { for all } a \in G\right\} \\
& =\left\{g \in G: g \in a H a^{-1} \text { for all } a \in G\right\} \\
& =\bigcap_{g \in G} g H g^{-1} .
\end{aligned}
$$

We now give two consequences of Proposition 3.2 that will be useful in our proof of Theorem 3.6. For $G \leq S_{n}$, let $G_{i}$ denote the point stabilizer of $i$ in $G$; that is, $G_{i}=\{g \in G: g(i)=i\}$.

Corollary 3.3. Let $G$ be a transitive subgroup of $S_{n}, G_{1}$ the point stabilizer of 1 in $G, L$ the left cosets of $G_{1}$ in $G$, and $\lambda$ the permutation representation of $G$ acting on $L$ by left multiplication. Then we have the following isomorphisms:

(1) $S_{L} \simeq S_{n}$, and

(2) $\lambda(G) \simeq G$.

Proof. To prove item (1), we show there is a well-defined bijection from $L$ to the set $\{1, \ldots, n\}$. Toward that end, let $\sigma \in G$ and suppose $\sigma(1)=i$. Then for every $a \in G_{1}$, we have $\sigma a(1)=i$, since $a(1)=1$. Thus every element in the coset $\sigma G_{1}$ sends 1 to $i$. Moreover, if $\tau \in G$ is any other element such that $\tau(1)=i$, then $\sigma^{-1} \tau(1)=\sigma^{-1}(i)=1$. This shows that $\sigma^{-1} \tau \in G_{1}$; i.e., that $\tau \in \sigma G_{1}$. Thus the map from $L$ to the set $\{1, \ldots, n\}$ is a well-defined injection. The map is surjective because $G$ is transitive. 


\section{CENTRALIZERS OF TRANSITIVE PERMUTATION GROUPS}

Therefore $\left[G: G_{1}\right]=n$, and it follows that $S_{L}$ is isomorphic to $S_{n}$ in a natural way.

To prove item (2), we show that Ker $\lambda$ is trivial. By Proposition 3.2, we have

$$
\text { Ker } \lambda=\bigcap_{g \in G} g G_{1} g^{-1} .
$$

For $g \in G$, if $g(1)=i$, then $g G_{1} g^{-1}=G_{i}$. Thus Ker $\lambda$ consists of those elements $g \in G$ such that $g(i)=i$ for every $i \in\{1, \ldots, n\}$. The only such element is the identity. This proves $\operatorname{Ker} \lambda$ is trivial, and therefore $\lambda(G) \simeq G$ by the First Isomorphism Theorem.

We now introduce another action on the left cosets of $H$. This action involves the normalizer $N(H)$ of $H$ in $G$.

Proposition 3.4. Let $G$ be a group, $H \leq G, N(H)$ the normalizer of $H$ in $G$, and $L$ the left cosets of $H$ in $G$. Then $N(H)$ acts on $L$ by the following action:

$$
n \cdot(a H)=a n^{-1} H .
$$

Let $\rho: N(H) \rightarrow S_{L}$ be the permutation representation of this action. Then $\operatorname{Ker} \rho=H$ and $\rho(N(H)) \simeq N(H) / H$.

Proof. First we show that $N(H)$ exhibits a well-defined action on $L$. Let $a, b \in G$ and suppose $a H=b H$. Then for all $n \in N(H)$,

$$
\begin{aligned}
n \cdot(a H) & =a n^{-1} H & & \\
& =a H n^{-1} & & \text { since } n \in N(H) \\
& =b H n^{-1} & & \text { since } a H=b H \\
& =b n^{-1} H & & \text { since } n \in N(H) \\
& =n \cdot(b H) . & &
\end{aligned}
$$

Thus the action is well-defined. Next, we have

$$
e \cdot(a H)=\left(a e^{-1}\right) H=a H .
$$

And if $x, y \in N(H)$, then we also have

$$
\begin{aligned}
x \cdot(y \cdot(a H)) & =x \cdot\left(a y^{-1} H\right) \\
& =a y^{-1} x^{-1} H \\
& =a(x y)^{-1} H \\
& =(x y) \cdot a H .
\end{aligned}
$$

So $N(H)$ does act on $L$. Let $\rho: N(H) \rightarrow S_{L}$ be the permutation representation of this action, so that $\rho(n)=\sigma_{n}$ where $\sigma_{n}(a H)=a n^{-1} H$. Then we

MISSOURI J. OF MATH. SCI., FALL 2015 
have,

$$
\begin{aligned}
\text { Ker } \rho & =\left\{n \in N(H): \sigma_{n}=\operatorname{id}_{L}\right\} \\
& =\left\{n \in N(H): \sigma_{n}(a H)=a H \text { for all } a \in G\right\} \\
& =\left\{n \in N(H): a n^{-1} H=a H \text { for all } a \in G\right\} \\
& =\left\{n \in N(H): n^{-1} \in H\right\} \\
& =H .
\end{aligned}
$$

The final statement in the proposition now follows from the First Isomorphism Theorem.

Our next result proves an important relationship between the two permutation representations $\lambda$ and $\rho$.

Proposition 3.5. Let $G$ be a group, $H \leq G, N(H)$ the normalizer of $H$ in $G$, and $L$ the left cosets of $H$ in $G$. Let $\lambda: G \rightarrow S_{L}$ and $\rho: N(H) \rightarrow S_{L}$ be the permutation representations in Propositions 3.2 and 3.4, respectively. Thus for a coset $a H$ of $H$, we have $\lambda(g)(a H)=g a H$ and $\rho(n)(a H)=$ $a n^{-1} H$ for all $g, a \in G$ and all $n \in N(H)$. Then

$$
C_{S_{L}}(\lambda(G))=\rho(N(H))
$$

where $C_{S_{L}}(\lambda(G))$ is the centralizer of $\lambda(G)$ in $S_{L}$.

Proof. First we show that $\rho(N(H)) \subseteq C_{S_{L}}(\lambda(G))$. Let $n \in N(H)$ and let $g \in G$ be arbitrary. We will show that $\rho(n) \lambda(g) \rho(n)^{-1}=\lambda(g)$ as permutations of $S_{L}$. Note that $\rho(n)^{-1}=\rho\left(n^{-1}\right)$ (see Definition 3.1). Then, for $a H \in L$, we have

$$
\begin{aligned}
\rho(n) \lambda(g) \rho\left(n^{-1}\right)(a H) & =\rho(n) \lambda(g)(\text { anH }) \\
& =\rho(n)(\operatorname{gan} H) \\
& =\operatorname{gann}^{-1} H \\
& =\operatorname{gaH} \\
& =\lambda(g)(a H) .
\end{aligned}
$$

Thus, $\rho(N(H)) \subseteq C_{S_{L}}(\lambda(G))$.

Conversely, let $\sigma \in C_{S_{L}}(\lambda(G))$ be arbitrary. Let $n \in G$ be defined by $\sigma(H)=n H$. Since $\sigma \in C_{S_{L}}(\lambda(G))$, this means $\sigma \lambda(a)=\lambda(a) \sigma$ for all $a \in G$. Evaluating both sides of the equation at $H$, we obtain:

$$
\sigma(a H)=a n H .
$$




\section{CENTRALIZERS OF TRANSITIVE PERMUTATION GROUPS}

If we can show $n \in N(H)$, then we are done since this proves $\sigma=\rho\left(n^{-1}\right) \in$ $\rho(N(H))$. Toward that end, let $h \in H$. Then we have

$$
\begin{aligned}
\sigma\left(n h n^{-1} H\right) & =n h n^{-1} n H \\
& =n h H \\
& =n H \\
& =\sigma(H) .
\end{aligned}
$$

Since $\sigma$ is a permutation, this implies $n h n^{-1} H=H$; i.e., $n h n^{-1} \in H$. Thus, $n \in N(H)$, as desired.

We are now able to prove our main result concerning automorphism groups of stem fields of polynomials.

Theorem 3.6. Let $f(x)$ be an irreducible degree $n$ polynomial defined over a field $F$. Let $\alpha_{1}, \ldots, \alpha_{n}$ be the roots of $f$ in some algebraic closure of $F$. Let $K=F\left(\alpha_{1}\right)$ be the stem field of $f$, and let $G$ be the Galois group of $f$ over $F$. Then Aut $(K / F) \simeq C_{S_{n}}(G)$, where $C_{S_{n}}(G)$ is the centralizer of $G$ in $S_{n}$. In particular, the order of the centralizer of $G$ in $S_{n}$ equals the number of roots of $f$ in $K$.

Proof. We first note that since $f$ is irreducible, $G$ is necessarily a transitive subgroup of $S_{n}$. Now let $G_{1}$ be the point stabilizer of 1 in $G$. Thus, $G_{1}$ corresponds to $K$ under the Galois correspondence of Theorem 2.3, since $G_{1}$ consists of those elements of $G$ that fix $\alpha_{1}$ (and hence, fix all of $K$ ). Let $L$ be the left cosets of $G_{1}$ in $G, N\left(G_{1}\right)$ the normalizer of $G_{1}$ in $G, \lambda$ and $\rho$ the permutation representations appearing in Propositions 3.2 and 3.4, respectively, and $C_{S_{L}}(\lambda(G))$ the centralizer of $\lambda(G)$ in $S_{L}$. Therefore, we have the following string of isomorphisms:

$$
\begin{aligned}
C_{S_{n}}(G) & \simeq C_{S_{L}}(\lambda(G)) & & \text { Corollary 3.3 } \\
& \simeq \rho\left(N\left(G_{1}\right)\right) & & \text { Proposition 3.5 } \\
& \simeq N\left(G_{1}\right) / G_{1} & & \text { Proposition 3.4 } \\
& \simeq \operatorname{Aut}(K / F) & & \text { Proposition 2.6. }
\end{aligned}
$$

The final sentence in the statement of the Theorem now follows, since the automorphisms of $K / F$ are in one-to-one correspondence with the roots of $f$ in $K$.

\section{Application: Quartic Galois Groups}

In this section, let $f(x)$ denote an irreducible quartic polynomial defined over the rational numbers and let $G$ be the Galois group of $f$. We give an algorithm for determining $G$ that does not involve factoring resolvent polynomials like the traditional approaches $[8,3]$. Though we are focusing

MISSOURI J. OF MATH. SCI., FALL 2015 


\section{AWTREY, N. MISTRY, AND N. SOLTZ}

TABle 1. Possible Galois groups of irreducible quartic polynomials.

\begin{tabular}{|c|c|c|c|c|}
\hline Name & Size & Generators & $|\mathbf{C}|$ & Parity \\
\hline$C_{4}$ & 4 & $(1234)$ & 4 & - \\
\hline$E_{4}$ & 4 & $(12)(34),(13)(24)$ & 4 & + \\
\hline$D_{4}$ & 8 & $(13),(1234)$ & 2 & - \\
\hline$A_{4}$ & 12 & $(123),(124)$ & 1 & + \\
\hline$S_{4}$ & 24 & $(12),(1234)$ & 1 & - \\
\hline
\end{tabular}

on the case where $f$ is defined over $\mathbf{Q}$, we point out that our method is valid over any field of characteristic different from two.

First we describe the possibilities for $G$. Since $f(x)$ is irreducible, $G$ can be identified with a transitive subgroup of $S_{4}$, well defined up to conjugation (different orderings of the roots correspond to conjugate subgroups). There are five conjugacy classes of transitive subgroups of $S_{4}$. Table 1 contains information on one representative group from each of these conjugacy classes, including the representative's Name, Size, and Generators. The names are standard: $C_{4}$ is the cyclic group of order $4, E_{4}$ is the elementary abelian group of order $4, C_{2} \times C_{2}$ (also called the Klein 4-group), $D_{4}$ is the dihedral group of order 8 , and $A_{4}$ and $S_{4}$ are the alternating and symmetric groups, respectively. The table also gives the order of the centralizer in $S_{4}$ in column $|\mathbf{C}|$ and the Parity of the group. The parity of a transitive subgroup $G \leq S_{n}$ is + if $G \leq A_{n}$ and - otherwise.

There is something important to observe about Table 1 . If we consider the two columns $|\mathbf{C}|$ and Parity, then no two groups have the same values for these two characteristics. This observation forms the core of our algorithm for computing the Galois group of an irreducible quartic polynomial (see Theorem 4.3). Before stating our algorithm, we introduce an important quantity associated to each polynomial.

Definition 4.1. Let $f(x)$ be a degree $n$ polynomial defined over a field $F$, and let $\alpha_{1}, \ldots, \alpha_{n}$ be the roots of $f$ in a fixed algebraic closure of $F$. The discriminant of $f$ is defined as

$$
\operatorname{Disc}(f)=\prod_{i<j}\left(\alpha_{i}-\alpha_{j}\right)^{2} .
$$

The discriminant of a polynomial is related to its Galois group in the following way. See [4, p. 610] for a proof.

Proposition 4.2. If $f(x)$ is a degree $n$ polynomial defined over a field $F$ with Galois group $G$, then Disc $(f)$ is a perfect square in $F$ if and only if $G \leq A_{n}$.

Furthermore, by the theory of elementary symmetric polynomials, it follows that $\operatorname{Disc}(f)$ can be expressed using only the coefficients of $f[4$, 


\section{CENTRALIZERS OF TRANSITIVE PERMUTATION GROUPS}

p. 611]. For example, in the case of quartic polynomials, [11] gives the following:

$$
\begin{aligned}
\operatorname{Disc}\left(x^{4}+a x^{3}+b x^{2}+c x+d\right)=- & 27 a^{4} d^{2}+2 a^{3} c\left(9 b d-2 c^{2}\right) \\
& -a^{2}\left(4 b^{3} d-b^{2} c^{2}-144 b d^{2}+6 c^{2} d\right) \\
& -2 a c\left(40 b^{2} d-9 b c^{2}+96 d^{2}\right) \\
& +4 b\left(4 b^{3}-b^{2} c^{2}-32 b d^{2}+36 c^{2} d\right) \\
& -27 c^{4}+256 d^{3} .
\end{aligned}
$$

The discriminant is used in the traditional approaches to computing Galois groups of quartic polynomials [8, 3]. We now give a brief description of each of these methods.

The approach taken in [8] involves computing and factoring a cubic polynomial that is related to the original quartic $f$. If this cubic polynomial is irreducible, the Galois group of $f$ is either $A_{4}$ or $S_{4}$, depending on whether the discriminant is a perfect square. In all other cases, the cubic polynomial has a rational root. If the cubic has three rational roots, then the Galois group is $E_{4}$. Otherwise, the cubic has an irreducible quadratic factor $g(x)$. The Galois group of $f$ is $C_{4}$ if $g(x)$ factors into two linear factors over the stem field of $x^{2}-\operatorname{Disc}(f)$, and it is $D_{4}$ otherwise.

The approach taken in [3] is more streamlined, as it involves only the discriminant of $f$ as well as computing and factoring a degree 6 polynomial $g(x)$ that is related to $f$. Let $L$ be the list of the degrees of the irreducible factors of $g(x)$. The Galois group of $f$ is $C_{4}$ if $L=\{1,1,4\}, E_{4}$ if $L=$ $\{2,2,2\}$, and $D_{4}$ if $L=\{2,4\}$. If $g(x)$ is irreducible, then the Galois group of $f$ is $A_{4}$ if $\operatorname{Disc}(f)$ is a perfect square and $S_{4}$ otherwise.

Our approach is similar to the one in [3]. We also make use of the discriminant of $f$, but instead of factoring a related degree 6 polynomial, we count the number of roots of $f$ in its stem field (which is equivalent to factoring $f$ over its stem field and counting linear factors).

Theorem 4.3. Let $f(x) \in \mathbf{Z}[x]$ be an irreducible quartic polynomial, and let $K$ be the stem field of $f$. Let \#A be the number of roots of $f$ in $K$, $D=\operatorname{Disc}(f)$, and $G$ the Galois group of $f$.

(1) If $\# A=4$, then $G=E_{4}$ if $D$ is a perfect square and $C_{4}$; otherwise.

(2) If $\# A=2$, then $G=D_{4}$.

(3) If $\# A=1$, then $G=A_{4}$ if $D$ is a perfect square and $S_{4}$; otherwise.

Proof. By Proposition 4.2, $D$ is a perfect square if and only if the parity of $G$ is + . Since the number of roots of $f$ in $K$ is equal to the size of $\operatorname{Aut}(K / \mathbf{Q})$, Theorem 3.6 therefore shows \# $A$ equals the order of the centralizer of $G$ in $S_{4}$. The validity of our algorithm now follows by examining the columns $|\mathbf{C}|$ and Parity in Table 1.

MISSOURI J. OF MATH. SCI., FALL 2015 


\section{AWTREY, N. MISTRY, AND N. SOLTZ}

Examples. To illustrate our algorithm, we compute the Galois groups of the following three polynomials: $A(x)=x^{4}+3 x+x, B(x)=x^{4}+5 x+5$, and $C(x)=x^{4}+7 x+7$. Let $\alpha, \beta$, and $\gamma$ denote a root of $A, B$, and $C$, respectively. Thus the stem field of $A$ is $\mathbf{Q}(\alpha)$, the stem field of $B$ is $\mathbf{Q}(\beta)$, and the stem field of $C$ is $\mathbf{Q}(\gamma)$. Note that we can factor a polynomial over its stem field using Algorithm 3.6.4 in [3].

There are two roots of $A(x)$ in its stem field; these are $\alpha$ and $\frac{1}{5}\left(4 \alpha^{3}-\right.$ $\left.2 \alpha^{2}+\alpha+9\right)$. According to Table $1, D_{4}$ is the only group with centralizer order 2. Thus the Galois group of $A(x)$ is $D_{4}$.

There are four roots of $B(x)$ in its stem field; these are $\beta,-\frac{1}{11}\left(4 \beta^{3}+\right.$ $\left.2 \beta^{2}+1 \beta+15\right),-\frac{1}{11}\left(4 \beta^{3}-9 \beta^{2}+12 \beta+15\right)$, and $\frac{1}{11}\left(8 \beta^{3}-7 \beta^{2}+2 \beta+30\right)$. Therefore the Galois group is either $C_{4}$ or $E_{4}$. Factoring the discriminant, we see that $\operatorname{Disc}(B(x))=5^{3} \cdot 11^{2}$, which is not a perfect square (because of the odd exponent of 5). Thus the Galois group of $B(x)$ is $C_{4}$.

There is only one root of $C(x)$ in its stem field; namely, $\gamma$. So the Galois group of $C(x)$ is either $A_{4}$ or $S_{4}$. Factoring the discriminant, we see that $\operatorname{Disc}(C(x))=7^{3} \cdot 67$, which is also not a square. We conclude that the Galois group of $C(x)$ is $S_{4}$.

\section{Application: Degree 15 5-Adic Fields}

Our final section deals with computing Galois groups of degree 15 polynomials defined over the field of 5-adic numbers. In the first subsection, we give a brief introduction to $p$-adic numbers $\mathbf{Q}_{p}$ and their extensions fields. Those interested in more details can find a good elementary account of $p$ adic numbers in [7]. Next, we describe the possible Galois groups of degree 15 polynomials defined over $\mathbf{Q}_{5}$ (see Table 2 ). For such a polynomial $f$, we then develop an algorithm for determining its Galois group $G$ when $G$ is a direct product of the form $H \times C_{3}$ where $H$ is a solvable transitive subgroup of $S_{5}$. Since the number of isomorphism classes of degree $n$ extensions of $\mathbf{Q}_{p}$ is finite [9, p. 54], we determine all isomorphism classes of degree 15 extensions of $\mathbf{Q}_{5}$ whose Galois group is of the form $H \times C_{3}$ (mentioned above). We find that there are 26 such extensions. We end with Tables 3 and 4 which give a defining polynomial for each extension along with a few of the extension's invariants.

5.1. Introduction to $p$-adic Numbers. The $p$-adic numbers are constructed from the rationals in much the same way the reals are constructed. In particular, consider the map $v_{p}: \mathbf{Q} \rightarrow \mathbf{Z} \cup\{\infty\}$ defined by

$$
v_{p}(x)= \begin{cases}n & \text { if } x=p^{n} a / b \text { with } p \nmid a b \\ \infty & \text { if } x=0 .\end{cases}
$$




\section{CENTRALIZERS OF TRANSITIVE PERMUTATION GROUPS}

The function $v_{p}$ is called the $p$-adic valuation and it gives rise to the $p$-adic absolute value $|\cdot|_{p}$ in the following way,

$$
|x|_{p}=\frac{1}{p^{v_{p}(x)}} \quad \text { for all } x \in \mathbf{Q} .
$$

The $p$-adic numbers are defined as the completion of $\mathbf{Q}$ with respect to this absolute value. The field $\mathbf{Q}_{p}$ has characteristic 0 and is a locally compact, totally disconnected Hausdorff topological space [7, p. 63].

The ring of $p$-adic integers $\mathbf{Z}_{p}$ is defined as the set $\left\{x \in \mathbf{Q}_{p}:|x|_{p} \leq 1\right\}$. The ring $\mathbf{Z}_{p}$ has a unique maximal ideal; namely, $p \mathbf{Z}_{p}$. It follows that every ideal of $\mathbf{Z}_{p}$ is of the form $p^{c} \mathbf{Z}_{p}$ for some integer $c$. Every element of $\mathbf{Q}_{p}$ can be written in the form $x / p^{n}$ for some $x \in \mathbf{Z}_{p}$ and some nonnegative integer $n$. Moreover, every element of $\mathbf{Z}_{p}$ can be represented uniquely as an infinite sum in "base $p$ " [7, p. 68]

$$
\mathbf{Z}_{p}=\left\{\sum_{k=0}^{\infty} a_{k} p^{k}: a_{k} \in \mathbf{Z} \text { with } 0 \leq a_{k} \leq p-1\right\} .
$$

Since $\mathbf{Q}_{p}$ has characteristic 0, the Primitive Element Theorem shows that an extension field of $\mathbf{Q}_{p}$ arises by adjoining the root of some monic irreducible polynomial over $\mathbf{Z}_{p}$ [4, p. 595]. By Krasner's Lemma [9, p. 43], this polynomial can be chosen to have integer coefficients. For an extension $K / \mathbf{Q}_{p}$ with $n=\left[K: \mathbf{Q}_{p}\right]$ and an element $x \in K$, let $g(y)=$ $y^{d}+a_{d-1} y^{d-1}+\cdots+a_{1} y+a_{0}$ be its minimal polynomial. We define the norm of $x$ from $K$ down to $\mathbf{Q}_{p}$ as,

$$
N_{K / \mathbf{Q}_{p}}(x)=(-1)^{n} g(0)^{n / d} .
$$

The norm is used to define an absolute value on $K$ that extends the $p$-adic absolute value on $\mathbf{Q}_{p}[7$, p. 151]. For $x \in K$, we define

$$
|x|=\sqrt[n]{\left|N_{K / \mathbf{Q}_{p}}(x)\right|_{p}}
$$

The absolute value on an extension $K$ gives rise to the corresponding valuation $v$ on $K$ by using the equation: $|x|=p^{-v(x)}$, where $v(0)=\infty$.

The valuation on $K$ is a homomorphism from the multiplicative group $K^{*}$ to the addivite group $\mathbf{Q}$. Its image is of the form $(1 / e) \mathbf{Z}$ where $e$ divides $\left[K: \mathbf{Q}_{p}\right]\left[7\right.$, p. 159]. We call $e$ the ramification index of $K / \mathbf{Q}_{p}$. The discriminant of $K$, denoted by $\operatorname{Disc}(K)$, is an ideal of $\mathbf{Z}_{p}$. We define the discriminant exponent of $K$ to be the integer $c$ such that $\operatorname{Disc}(K)=p^{c} \mathbf{Z}_{p}$.

5.2. Galois Groups of Degree 15 5-adic Fields. If $f(x) \in \mathbf{Z}_{5}[x]$ is an irreducible polynomial of degree 15, then it follows that the Galois group $G$ of $f$ over $\mathbf{Q}_{p}$ is a transitive subgroup of $S_{15}$, of which there are 104 . However, the arithmetic in extensions of $\mathbf{Q}_{p}$ is specialized, and this limits the possibility for what $G$ can be.

MISSOURI J. OF MATH. SCI., FALL 2015 


\section{AWTREY, N. MISTRY, AND N. SOLTZ}

TABLE 2. The 24 transitive subgroups of $S_{15}$ that are possible Galois groups of degree 15 polynomials defined over $\mathbf{Q}_{5}$.

\begin{tabular}{|c|c|c|c|}
\hline T & Size & $|\mathbf{C}|$ & Parity \\
\hline 1 & 15 & 15 & + \\
\hline 2 & 30 & 1 & - \\
\hline 3 & 30 & 3 & + \\
\hline 4 & 30 & 5 & - \\
\hline 6 & 60 & 1 & + \\
\hline 7 & 60 & 1 & - \\
\hline 8 & 60 & 3 & - \\
\hline 9 & 75 & 5 & + \\
\hline 11 & 120 & 1 & - \\
\hline 12 & 150 & 1 & + \\
\hline 13 & 150 & 5 & - \\
\hline 14 & 150 & 1 & - \\
\hline 17 & 300 & 1 & + \\
\hline 18 & 300 & 1 & - \\
\hline 19 & 300 & 1 & - \\
\hline 25 & 375 & 5 & + \\
\hline 27 & 600 & 1 & - \\
\hline 30 & 750 & 1 & + \\
\hline 31 & 750 & 1 & - \\
\hline 32 & 750 & 5 & - \\
\hline 37 & 1500 & 1 & + \\
\hline 38 & 1500 & 1 & - \\
\hline 40 & 1500 & 1 & - \\
\hline 49 & 3000 & 1 & - \\
\hline
\end{tabular}

For example, in [1, Lemma 2.2] it is shown that $G$ must have the following properties:

- $G$ contains a normal subgroup $I$ such that $G / I$ is cyclic.

- $I$ contains a normal subgroup $W$ such that $W$ is a 5 -group (possibly trivial).

- $I / W$ is cyclic of order dividing $5^{[G: I]}-1$.

Using the software GAP [6], direct computation on the 104 transitive subgroups of $S_{15}$ shows that only 24 groups are possible Galois groups of degree 15 polynomials over $\mathbf{Q}_{5}$. We identify these groups in Table 2 using the transitive numbering system in [6]. Specifically, an entry of $j$ in column $\mathbf{T}$ refers to the group TransitiveGroup $(15, j)$ in GAP. The table also includes the Size of each group, the order of its centralizer in $S_{15}$ (in column $|\mathbf{C}|$ ), and its Parity.

Notice that one group in Table 2 has a centralizer order of 15 and two have a centralizer order of 3 . These three groups are $\mathrm{T} 1=C_{15}, \mathrm{~T} 3=$ 


\section{CENTRALIZERS OF TRANSITIVE PERMUTATION GROUPS}

$D_{5} \times C_{3}$, and $\mathrm{T} 8=F_{5} \times C_{3}$. Here $F_{5}$ is the Frobenius group of order 20 ; it is a semidirect product $C_{5} \rtimes C_{4}$, generated by (12345) and (2354). Note that these three groups are of the form $H \times C_{3}$ where $H$ is a solvable transitive subgroup of $S_{5}$.

The group T1 is the only group among the 24 whose centralizer in $S_{15}$ has 15 elements. Similarly, T3 and T8 are the only groups whose centralizers have 3 elements, and these two groups have different parities. We have therefore proven the following theorem, which determines when a degree 15 polynomial defined over $\mathbf{Q}_{5}$ has a Galois group among T1, T3, and T8.

Theorem 5.1. Let $f(x)$ be an irreducible degree 15 polynomial defined over the 5-adic numbers, and let $K$ be the stem field of $f$. Let \#A be the number of roots of $f$ in $K, D=\operatorname{Disc}(f)$, and $G$ the Galois group of $f$.

(1) If $\# A=15$, then $G=C_{15}$.

(2) If $\# A=3$, then $G=D_{5} \times C_{3}$ if $D$ is a perfect square and $F_{5} \times C_{3}$ otherwise.

Since the number of degree 15 extensions of $\mathbf{Q}_{5}$ is finite [9, p. 54], we can count how many extensions have Galois group $C_{15}, D_{5} \times C_{3}$, and $F_{5} \times C_{3}$. First, we use [12] to compute polynomials defining all nonisomorphic degree 15 extension of $\mathbf{Q}_{5}$. There are 1012 such polynomials. For each polynomial, we compute the number of roots of that polynomial in its stem field. This is possible using the $p$-adic root-finding algorithm in [10]. We extract those polynomials whose stem field contains either 15 roots or 3 roots. If the stem field contains 15 roots, then the Galois group is $C_{15}$.

Otherwise, we compute the polynomial's discriminant and determine if it is a perfect square in $\mathbf{Q}_{5}$. This is a straightforward task due to Hensel's Lemma [7, p. 71]. In particular, we write $D=p^{k} u$ where $\operatorname{gcd}(p, u)=1$. Then $D$ is a perfect square in $\mathbf{Q}_{5}$ if and only if $k$ is even and $u$ is a quadratic residue modulo 5 . If the discriminant is a perfect square, then $G=D_{5} \times C_{3}$. Otherwise $G=F_{5} \times C_{3}$.

Of the 1012 polynomials defining nonisomorphic degree 15 extensions of $\mathbf{Q}_{5}$, six have $C_{15}$ as Galois group, three have $D_{5} \times C_{3}$ as Galois group, and 17 have $F_{5} \times C_{3}$ as Galois group. Tables 3 and 4 list these 26 polynomials, their Galois group, and the ramification index $e$ and discriminant exponent $c$ of their stem field.

\section{ACKNOWLEDGEMENTS}

The authors were supported in part by NSF grant \#DMS-1148695. The authors would like to thank the anonymous reviewer for their close reading and helpful comments, Elon University for supporting this project, and the Center for Undergraduate Research in Mathematics for their support.

MISSOURI J. OF MATH. SCI., FALL 2015 


\section{AWTREY, N. MISTRY, AND N. SOLTZ}

TABle 3. Polynomials over $\mathbf{Q}_{5}$ whose Galois group is T1 or T3. Also included are the extension's ramification index e and discriminant exponent $\mathbf{c}$. The table is sorted first by Galois group, then by ramification index, then by discriminant exponent.

\begin{tabular}{|l|l|l|l|}
\hline Polynomials & e & c & G \\
\hline
\end{tabular}

\begin{tabular}{|c|c|c|c|}
\hline$x^{15}+x^{2}+2$ & 1 & 0 & $\mathrm{~T} 1$ \\
\hline $\begin{array}{c}x^{15}+585 x^{14}+505 x^{13}+370 x^{12}+165 x^{11}+378 x^{10}+395 x^{9}+170 x^{8} \\
+315 x^{7}+95 x^{6}+306 x^{5}+315 x^{4}+260 x^{3}+5 x^{2}+260 x+607\end{array}$ & 5 & 24 & $\mathrm{~T} 1$ \\
\hline $\begin{array}{c}x^{15}+405 x^{14}+120 x^{13}+10 x^{12}+90 x^{11}+188 x^{10}+135 x^{9}+395 x^{8} \\
+505 x^{7}+345 x^{6}+341 x^{5}+300 x^{4}+95 x^{3}+75 x^{2}+470 x+457\end{array}$ & 5 & 24 & $\mathrm{~T} 1$ \\
\hline $\begin{array}{c}x^{15}+380 x^{14}+555 x^{13}+575 x^{12}+160 x^{11}+43 x^{10}+70 x^{9}+125 x^{8} \\
+330 x^{7}+170 x^{6}+546 x^{5}+305 x^{4}+75 x^{3}+95 x^{2}+370 x+382\end{array}$ & 5 & 24 & $\mathrm{~T} 1$ \\
\hline $\begin{array}{c}x^{15}+70 x^{14}+315 x^{13}+80 x^{12}+530 x^{11}+518 x^{10}+565 x^{9}+425 x^{8} \\
+480 x^{7}+30 x^{6}+386 x^{5}+275 x^{4}+5 x^{3}+295 x^{2}+100 x+132\end{array}$ & 5 & 24 & $\mathrm{~T} 1$ \\
\hline $\begin{array}{c}x^{15}+315 x^{14}+285 x^{13}+45 x^{12}+425 x^{11}+133 x^{10}+620 x^{9}+325 x^{8} \\
+365 x^{7}+365 x^{6}+516 x^{5}+35 x^{4}+575 x^{3}+275 x^{2}+420 x+257\end{array}$ & 5 & 24 & $\mathrm{~T} 1$ \\
& & \\
\hline
\end{tabular}

\begin{tabular}{|c|c|c|c|}
\hline $\begin{aligned} x^{15} & +90 x^{14}+15 x^{13}+70 x^{12}+110 x^{11}+60 x^{10}+85 x^{9}+110 x^{8}+5 x^{7} \\
& +15 x^{6}+14 x^{5}+15 x^{4}+75 x^{3}+90 x^{2}+20 x+43\end{aligned}$ & 5 & 18 & T3 \\
\hline $\begin{aligned} x^{15} & +70 x^{14}+100 x^{13}+85 x^{12}+70 x^{11}+30 x^{10}+85 x^{9}+85 x^{8}+80 x^{7} \\
& +20 x^{6}+49 x^{5}+105 x^{4}+5 x^{3}+115 x^{2}+80 x+83\end{aligned}$ & 5 & 18 & T3 \\
\hline $\begin{aligned} x^{15} & +445 x^{14}+130 x^{13}+265 x^{12}+560 x^{11}+323 x^{10}+505 x^{9}+545 x^{8} \\
& +280 x^{7}+580 x^{6}+306 x^{5}+120 x^{4}+75 x^{3}+175 x+252\end{aligned}$ & 5 & 24 & T3 \\
\hline
\end{tabular}

\section{REFERENCES}

1. C. Awtrey, N. Miles, J. Milstead, C. Shill, and E. Strosnider, Degree 14 2-adic fields, Involve, 8.2 (2015), 329-336. MR 3320863.

2. C. Awtrey and E. Strosnider, A linear resolvent for degree 14 polynomials, Collaborative Mathematics and Statistics Research: Topics from the 9th Annual UNCG Regional Mathematics in Statistics Conference, Springer Proceedings of Mathematics \& Statistics, Vol. 109, Springer, New York, 2015.

3. H. Cohen, A course in computational algebraic number theory, Graduate Texts in Mathematics, Vol. 138, Springer-Verlag, Berlin, 1993. MR 1228206 (94i:11105).

4. D. S. Dummit and R. M. Foote, Abstract Algebra, third ed., John Wiley \& Sons, Inc., Hoboken, NJ, 2004. MR 2286236 (2007h:00003).

5. C. Ficker and J. Klüners, Computation of Galois groups of rational polynomials, LMS J. Comput. Math., 17.1 (2014), 141-158. MR 3230862.

6. The GAP Group, GAP - Groups, Algorithms, and Programming, Version 4.4.12, 2008.

7. F. Q. Gouvêa, p-adic numbers, second ed., Universitext, Springer-Verlag, Berlin, 1997, An introduction. MR 1488696 (98h:11155).

8. L.-C. Kappe and B. Warren, An elementary text for the Galois group of a quartic polynomial, Amer. Math. Monthly, 96.2 (1989), 133-137. MR 992075 (90i:12006). 


\section{CENTRALIZERS OF TRANSITIVE PERMUTATION GROUPS}

TABLE 4. Polynomials over $\mathbf{Q}_{5}$ whose Galois group is T8.

This is a continuation of Table 3 .

\section{Polynomials}

\begin{tabular}{|l|l|l|}
\hline & $\mathbf{c}$ & $\mathbf{G}$ \\
\hline
\end{tabular}

\begin{tabular}{|c|c|c|c|}
\hline$x^{15}+20 x^{11}+22 x^{10}+5 x^{9}+15 x^{5}+10 x^{4}+10 x^{3}+5 x^{2}+15 x+3$ & 5 & 15 & T8 \\
\hline $\begin{aligned} x^{15} & +15 x^{13}+10 x^{12}+5 x^{11}+12 x^{10}+5 x^{9}+5 x^{7}+10 x^{6}+10 x^{5}+10 x^{4} \\
& +5 x^{3}+20 x^{2}+15 x+3\end{aligned}$ & 5 & 15 & T8 \\
\hline $\begin{array}{l}x^{15}+20 x^{14}+15 x^{12}+5 x^{11}+2 x^{10}+20 x^{9}+10 x^{8}+20 x^{7}+20 x^{6} \\
\quad+15 x^{5}+15 x^{4}+10 x^{3}+5 x^{2}+3\end{array}$ & 5 & 15 & $\overline{\mathrm{T} 8}$ \\
\hline $\begin{array}{l}x^{15}+10 x^{14}+20 x^{13}+15 x^{12}+20 x^{11}+17 x^{10}+15 x^{8}+20 x^{7}+10 x^{6} \\
+20 x^{5}+15 x^{4}+15 x^{3}+15 x^{2}+20 x+18\end{array}$ & 5 & 15 & T8 \\
\hline $\begin{aligned} x^{15} & +90 x^{14}+100 x^{13}+80 x^{12}+110 x^{11}+15 x^{10}+5 x^{9}+35 x^{8}+45 x^{7} \\
& +90 x^{6}+34 x^{5}+30 x^{4}+100 x^{3}+55 x^{2}+60 x+38\end{aligned}$ & 5 & 18 & Г8 \\
\hline $\begin{aligned} x^{15} & +85 x^{14}+90 x^{13}+85 x^{11}+115 x^{10}+10 x^{9}+20 x^{8}+40 x^{7}+55 x^{6} \\
& +99 x^{5}+120 x^{4}+120 x^{3}+90 x^{2}+75 x+8\end{aligned}$ & 5 & 18 & $\mathrm{~T} 8$ \\
\hline $\begin{aligned} x^{15} & +95 x^{14}+75 x^{13}+65 x^{12}+45 x^{11}+60 x^{10}+25 x^{9}+70 x^{8}+65 x^{7} \\
& +75 x^{6}+23 x^{5}+55 x^{3}+60 x^{2}+115 x+83\end{aligned}$ & 5 & 21 & $\mathrm{~T} 8$ \\
\hline $\begin{aligned} x^{15} & +15 x^{14}+10 x^{13}+95 x^{12}+45 x^{11}+10 x^{10}+95 x^{9}+95 x^{8}+70 x^{7} \\
& +30 x^{6}+18 x^{5}+110 x^{4}+50 x^{3}+35 x^{2}+70 x+123\end{aligned}$ & 5 & 21 & $\overline{78}$ \\
\hline $\begin{aligned} x^{15} & +85 x^{14}+40 x^{13}+25 x^{12}+50 x^{11}+50 x^{10}+40 x^{9}+95 x^{8}+90 x^{7} \\
& +105 x^{6}+103 x^{5}+70 x^{4}+115 x^{3}+100 x^{2}+45 x+43\end{aligned}$ & 5 & 21 & T8 \\
\hline $\begin{aligned} x^{15} & +100 x^{14}+55 x^{13}+40 x^{12}+40 x^{11}+70 x^{10}+95 x^{9}+5 x^{8}+110 x^{7} \\
& +45 x^{6}+73 x^{5}+10 x^{4}+80 x^{3}+55 x^{2}+10 x+103\end{aligned}$ & 5 & 21 & T8 \\
\hline $\begin{aligned} x^{15} & +605 x^{14}+220 x^{13}+130 x^{12}+380 x^{11}+443 x^{10}+410 x^{8}+235 x^{7} \\
& +485 x^{6}+171 x^{5}+390 x^{4}+150 x^{3}+600 x^{2}+60 x+522\end{aligned}$ & 5 & 24 & 8 \\
\hline $\begin{aligned} x^{15} & +130 x^{14}+605 x^{12}+200 x^{11}+213 x^{10}+140 x^{9}+435 x^{8}+305 x^{7} \\
& +620 x^{6}+131 x^{5}+190 x^{4}+560 x^{3}+560 x^{2}+445 x+512\end{aligned}$ & 5 & 24 & T8 \\
\hline $\begin{aligned} x^{15} & +570 x^{14}+530 x^{13}+620 x^{12}+70 x^{11}+549 x^{10}+460 x^{9}+490 x^{8} \\
& +615 x^{7}+595 x^{6}+34 x^{5}+60 x^{4}+100 x^{3}+300 x^{2}+620 x+537\end{aligned}$ & 5 & 27 & $\mathrm{~T} 8$ \\
\hline $\begin{aligned} x^{15} & +470 x^{14}+555 x^{13}+595 x^{12}+370 x^{11}+54 x^{10}+335 x^{9}+65 x^{8} \\
& +440 x^{7}+395 x^{6}+449 x^{5}+260 x^{4}+575 x^{3}+200 x^{2}+20 x+7\end{aligned}$ & 5 & 27 & T8 \\
\hline $\begin{aligned} & x^{15}+420 x^{14}+305 x^{13}+45 x^{12}+170 x^{11}+594 x^{10}+160 x^{9}+65 x^{8} \\
&+190 x^{7}+95 x^{6}+194 x^{5}+35 x^{4}+375 x^{3}+525 x^{2}+495 x+367 \\
&\end{aligned}$ & 5 & 27 & T8 \\
\hline $\begin{aligned} x^{15} & +170 x^{14}+430 x^{13}+320 x^{12}+420 x^{11}+519 x^{10}+10 x^{9}+590 x^{8} \\
& +290 x^{7}+45 x^{6}+514 x^{5}+385 x^{4}+300 x^{3}+200 x^{2}+420 x+202\end{aligned}$ & 5 & 27 & T8 \\
\hline $\begin{aligned} x^{15} & +620 x^{14}+455 x^{13}+95 x^{12}+320 x^{11}+4 x^{10}+210 x^{9}+590 x^{8} \\
& +490 x^{7}+295 x^{6}+489 x^{5}+110 x^{4}+225 x^{3}+175 x^{2}+495 x+1\end{aligned}$ & 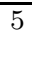 & 27 & T8 \\
\hline
\end{tabular}

9. S. Lang, Algebraic Number Theory, second ed., Graduate Texts in Mathematics, Vol. 110, Springer-Verlag, New York, 1994. MR 1282723 (95f:11085).

10. P. Panayi, Computation of Leopoldt's p-adic regulator, Ph.D. thesis, University of East Anglia, December 1995.

11. The PARI Group, PARI/GP - Computational Number Theory, version 2.3.4, 2008, available from http://pari.math.u-bordeaux.fr/.

12. S. Pauli and X.-F. Roblot, On the computation of all extensions of a p-adic field of a given degree, Math. Comp., 70.236 (2001), 1641-1659 (electronic). MR 1836924. (2002e:11166)

MISSOURI J. OF MATH. SCI., FALL 2015 


\section{AWTREY, N. MISTRY, AND N. SOLTZ}

13. L. Soicher and J. McKay, Computing Galois group over the rationals, J. Number Theory, 20.3 (1985), 273-281. MR 797178 (87a:12002).

14. R. P. Stauduhar, The determination of Galois groups, Math. Comp., 27 (1973), 981-996. MR 0327712 (48 \#6054).

MSC2010: 20B35, 12Y05, 11R32, 11S20

Key words and phrases: Galois group computation; centralizers; normalizers; $p$-adic fields; quartic extensions

Elon University, Campus Box 2320, Elon, NC 27244

E-mail address: cawtrey@elon.edu

Department of Mathematics, Mathematics Building, University of Maryland, College Park, MD 20742

E-mail address: nmistry@elon.edu

Department of Mathematics and Statistics, Elon University, Campus Box 5814, ELON, NC 27244

E-mail address: nsoltz@elon.edu 